João Lameu da Silva Júnior ${ }^{1}$

Victória Anselmo Haddad²

Osvaldir Pereira Taranto ${ }^{2}$

Harrson Silva Santana2, *

\title{
Design and analysis of new micromixers based on distillation column trays
}

\section{Abstract Text}

In the present paper four passive micromixers designs (G1, G2, G3 and G4) inspired on distillation columns trays were proposed. The devices performance was assessed by numerical simulations. The mixing performance was investigated for a Reynolds number range from 0.01 to 100 and channel height of 200 $\mu \mathrm{m}, 500 \mu \mathrm{m}$ and $1000 \mu \mathrm{m}$ for oil/ethanol flow. G1 and G4 designs provided high mixing index (>0.975). The $\mathrm{G} 1$ device achieved superior mixing performance with a moderate pressure drop (about $0.5 \mathrm{MPa}$ ) due to the induced flow recirculation pattern for a relative high flow rate of $0.21 \mathrm{~L} / \mathrm{min}$, highlighting the potential use of such microdevice for scale-up and numbering-up of microdevices in modular chemical plant processing.

Keywords: chaotic advection; microfluidics; micromixer; numerical simulation; process intensification

\section{Author affiliations}

${ }^{1}$ Universidade Federal do ABC, CECS - Centro de Engenharia, Modelagem e Ciências Sociais, Alameda da Universidade, s/n., 09606-045, São Bernardo do Campo, SP, Brazil.

${ }^{2}$ University of Campinas, School of Chemical Engineering, Albert Einsten Av. 500, 13083-852, Campinas, SP, Brazil.

Email corresponding author:

harrison.santana@gmail.com; harrson@unicamp.br

\section{Introduction}

Microfluidics is the science and technology that handles fluid flow in micrometric scale structures. Recently, microfluidic devices received great attention due to its easiness of handling continuous flow and great precision control over process variables (e.g. temperature), requirement of low amounts of reactants and samples, high selectivity and product yield in short residence times, high surface area to volume ratio, enhancement of heat and mass transfer, safety operation of flammable, toxic and explosive substances [1-3]. These characteristics allowed the use of microdevices in different fields, such as food and agriculture [4], biomedical systems [5-8], heat exchanger [9-11], microseparators [12] and microreactors [13-15].

Microreactors have been used in distinct chemical and biochemical reactive systems, including organic synthesis [16], biotechnology process [17], water purification [18] and biodiesel synthesis $[14,19,20]$. Such devices are characterized by micrometric channels where the reactants flow and are contacted. As a consequence of the size scale, usually the operating conditions results in laminar flow regime marked by the absence of macromixing of fluid layers. Accordingly, molecular diffusion plays a fundamental role in the process kinetics and long channels or residence times are required to complete the mixing and the reaction [21]. Micromixers appear as an interesting alternative to increase mixing 
efficiency and consequently to reduce the reaction time, and can be grouped in two types: active and passive [22.23].

Active micromixers use external energy sources, e.g., electrical or magnetic fields, while passive employs the own flow energy from pumping system. Passive devices present easier manufacturing procedure with smaller costs. However, such devices requires longer longitudinal lengths and operate under higher Reynolds numbers, and consequently under high pressure drops, regarding active mixers. The increment of surface area to volume ratio by the use of internal obstacles results in mass transfer enhancement by several mechanisms including diffusion path reduction, split and recombination of streams, vortex generation and chaotic advection. In this context, an optimal micromixer design must encompass some of these mechanisms to provide the superior mixing degree per unit of pressure drop.

Mass transfer intensification is one of the key problems in chemical engineering and distillation unit operation, i.e., sharp separation of liquid mixture in almost pure compounds by volatility difference, is a classical and well-succeeded example, with constant design innovation of columns in order to enhance the mass transfer of the species. The present paper aims to approach these two research fields proposing new passive micromixer based on distillation column tray layouts, including designs inspired on traditional plate and a Vortex Tray (Sulzer ${ }^{\circledR}$ ). 3D CAD modeling aligned with Computational Fluid Dynamics (CFD) simulation were employed and the micromixer performance was evaluated by mixing degree of oil and ethanol for a Reynolds number range of 0.01-100.

\section{Micromixer Design and Numerical Investigation}

\subsection{Micromixer Design and Performance Assessment}

The 3D geometries were created in Autodesk Inventor (2017). Four distinct layouts (Figure 1) were designed. Figure 1 shows the micromixer geometric details. All proposed design was inspired in a 2D perspective of a column tray. The microdevices are composed by 17 micromixer units arranged in series, resulting in $35 \mathrm{~mm}$-long channels. A micromixer unit is defined as a set of static elements (Figure 1). The design present in Figure $1 \mathrm{a}, \mathrm{b}$ (geometry $\mathrm{G} 1$ ) was based in a two-pass tray composed by a central weir and two lateral weirs alternatively disposed along the channel. The design presented in Figure 1c (geometry G2) is similar of Figure $1 \mathrm{~b}$, with the variation of thin fluid-passages through the central weir, representing a perforated plate from distillation columns. Figure 1d (geometry G3) and 1e (geometry G4) were inspired in Sulzer ${ }^{\circledast}$ Vortex Tray that employs a conical downcomer. The proposed design consisted in conical weirs with an outlet cap due to the 2D projection. The design represented in Figure 1e possesses additional baffles to direct the flow only through the $2 \mathrm{D}$ conical weirs.

The height of the micromixer channels, $H$, varied of $200 \mu \mathrm{m}, 500 \mu \mathrm{m}$ and $1000 \mu \mathrm{m}$. The main channel width and longitudinal lengths were assigned to $1.5 \mathrm{~mm}$ and $35 \mathrm{~mm}$, respectively. Figure 2 presents the geometric parameters based on the characteristic length, $L_{c}=500 \mu \mathrm{m}$. All geometries present Y-shape inlets. The software ANSYS ICEM 17 were used to generate the numerical meshes. The ANSYS CFX 17 was used to assess the micromixers performance quantified by mixing and performance indexes. 
(a)

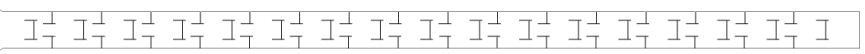

(b)

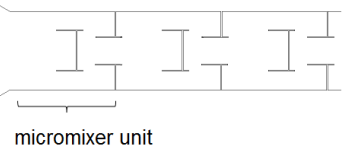

(d)

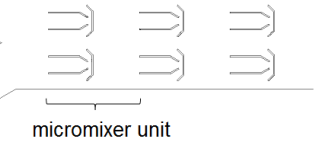

(c)

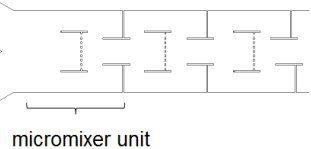

(e)

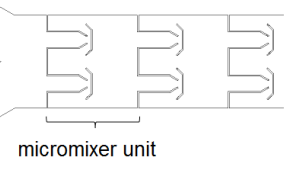

Figure 1. Details of the four micromixer designs created in Autodesk Inventor: (a) complete micromixer channel for geometry G1; details of internal passive/static mixers of geometries: (b) G1; (c) G2; (d) G3; (e) G4.

(a)

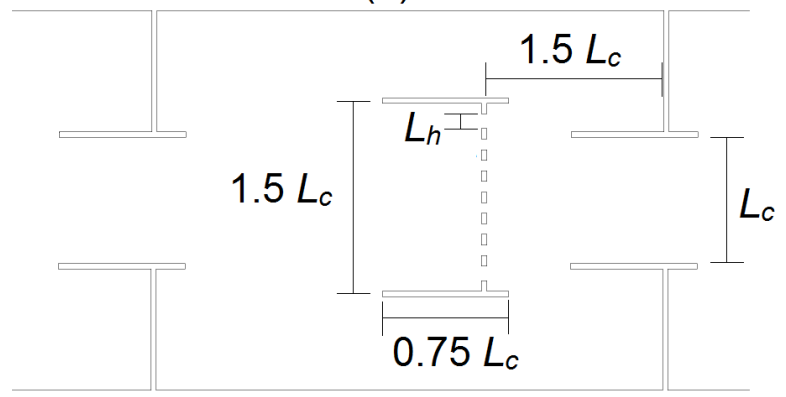

(b)

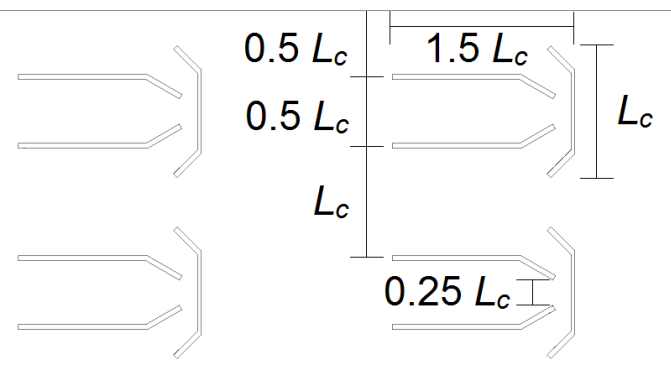

Figure 2. Geometric parameters of the proposed designs: (a) geometries $G 1$ and $G 2$ (with $L_{h}=0.06 L_{c}$ ) (b) geometries G3 e G4. The characteristic parametric length is $L_{c}=500 \mu \mathrm{m}$.

The fluid mixing evaluation was carried out with oil-ethanol flow, once these fluids are employed in biodiesel synthesis and present low miscibility in addition to the high viscosity of the oil phase, hindering the mass transfer process. The micromixer performance was evaluated by mixing and performance indexes. The mixing degree between sunflower oil and the ethanol was determined based on the mass fraction standard deviation of one in cross sections normal to the flow direction (Equation 1): 


$$
\sigma=\sqrt{\frac{\sum\left(Y_{i}-\bar{Y}\right)^{2}}{N}}
$$

where $\sigma$ is the mass fraction variance, $Y_{i}$ is the mass fraction at sampling point $i, \bar{Y}$ is the mass fraction average and $N$ is the number of sampling points in the cross section. Then, the mixing index was calculated from Equation 2:

$$
M=1-\sqrt{\frac{\sigma^{2}}{\sigma_{\max }^{2}}}
$$

where $M$ is the mixing index and $\sigma_{\max }^{2}$ is the maximum variance over the data interval. The mixing index varies from zero (complete fluids segregation) to a unity (complete or ideal fluid mixing).

The Performance Index $(P I)$ is defined as the ratio of mixing index to the unit pressure drop required, according to Equation 3. Accordingly, efficient micromixers should provide high mixing index with lower pressure drop [14,27].

$$
P I=\frac{M}{\Delta P}
$$

where $\Delta P$ is the pressure drop along the mixing channel.

\subsection{Mathematical Model}

The fluid dynamic studie were accomplished using the computational code ANSYS CFX 17.0. The mathematical model considerations were: incompressible, steady-state, isothermal and laminar flow conditions. Therefore, the conservation of total mass (continuity) (Equation 4), momentum (Navier-Stokes) (Equation 5) and mass of chemical species (Equations 6 and 7) were numerically solved:

$$
\begin{aligned}
& \nabla \cdot U=0 \\
& \rho(U \cdot \nabla U)=-\nabla P+\mu \nabla^{2} U+\rho g \\
& \rho\left(U \cdot \nabla Y_{A}\right)=\rho D_{A B} \nabla^{2} Y_{A} \\
& Y_{B}=1-Y_{A}
\end{aligned}
$$

where $\rho$ is the specific mass $\left(\mathrm{kg} \mathrm{m}^{-3}\right), U$ is the velocity vector $\left(\mathrm{m} \mathrm{s}^{-1}\right), \mu$ is the dynamic viscosity (Pa s), $g$ is the gravity acceleration $\left(\mathrm{m} \mathrm{s}^{-2}\right), P$ is the pressure $\left(\mathrm{kg} \mathrm{m}^{-1} \mathrm{~s}^{-2}\right), Y$ is the mass fraction, $D$ is the mass transfer diffusion coefficient $\left(\mathrm{m}^{2} \mathrm{~s}^{-1}\right)$, estimated using the Wilke-Chang correlation [45], considering ethanol (chemical species A) in sunflower oil (chemical species B).

The boundary conditions employed in the numerical solution of transport equations are described below:

- Alcohol feed stream (Inlet 1): pure alcohol flowing into domain $\left(Y_{A}=1 ; Y_{T G}=0\right.$ or $\left.Y_{W}=0\right)$, with uniform velocity normal to boundary inlet surface;

- Sunflower oil feed stream or water feed stream (Inlet 2): pure oil (triglyceride) or water stream flowing into domain $\left(Y_{T G}=1\right.$ or $\left.Y_{W}=1 ; Y_{A}=0\right)$, with uniform velocity normal to boundary inlet surface. Both inlet velocities were defined based on Reynolds number (Equation 8) and ethanol/oil molar ratio.

$\operatorname{Re}=\frac{\rho \bar{U} D_{h}}{\mu}$

where $\bar{U}$ is the mean velocity $\left(\mathrm{m} \mathrm{s}^{-1}\right), D_{H}$ is the hydraulic diameter $(\mathrm{m}), \mu$ is the dynamic viscosity $\left(\mathrm{kg} \mathrm{m}^{-1}\right.$ $\left.\mathrm{s}^{-1}\right)$, and $\rho$ is the fluid specific mass $\left(\mathrm{kg} \mathrm{m}^{-3}\right)$ 
- Microchannel outlet (Outlet): zero relative average pressure;

- Microchannel walls (Walls): no-slip condition at solid surfaces.

In the numerical solution, high order discretization schemes were employed. A convergence criteria of RMS = $1 \times 10^{-6}$ was defined for a solving iteration range of 500-5000. The simulations were solved in parallel processing in computer nodes with 8 Intel Xeon 3GHz, 16GB RAM processors with Linux Suse 64-bit OS.

\section{Results and Discussion}

\subsection{Mesh Independence Analysis}

Numerical mesh tests are fundamental to ensure numerical predictions with minimal effects of spatial discretization errors in the results [14]. The more complex design, geometry $\mathrm{G} 4$ (with the channel height of $1000 \mu \mathrm{m}$ ), was used in the numerical independence analysis. Numerical grids were generated with different refinement levels, from $1 \times 10^{6}$ to $5.7 \times 10^{6}$ of elements. This first analysis was performed for a Reynolds number of 100 for oil-ethanol system. The mixing index and pressure drop was used in the spatial discretization independence evaluation. The results are shown in Figure 2 and Table 1.

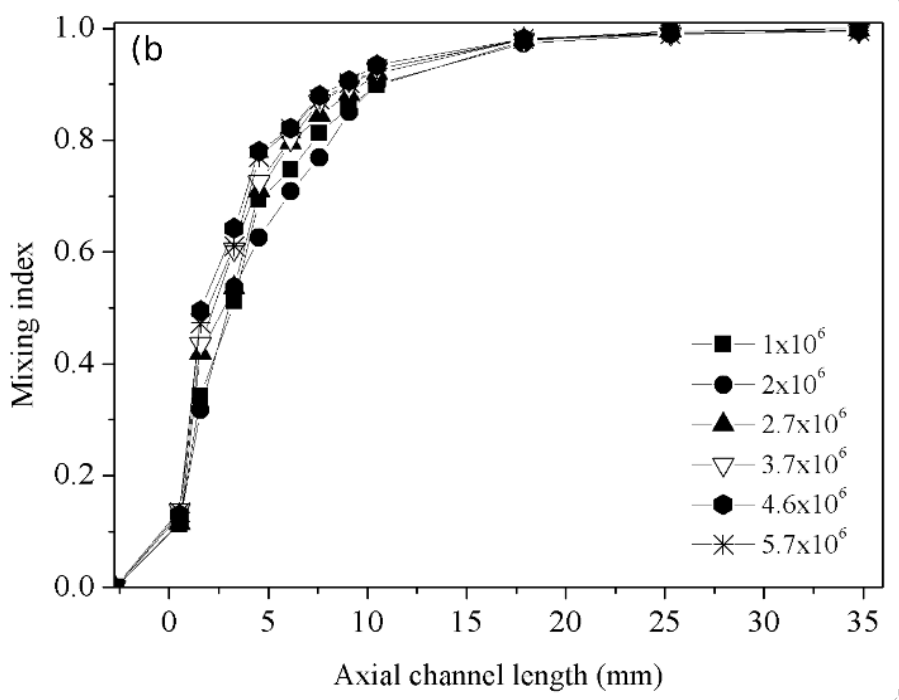

Figure 2. Independence mesh test result: mixing index for vegetable oil-ethanol flow at $R e=100$.

From Figure 2 is noticed that all grid refinements presented similar behavior for the chemical species distribution along the microchannels. Accordingly, the independence mesh analysis must be verified by the pressure drop predictions by the different grid refinements. Table 1 presents the pressure drops and the relative deviation taking as reference the most refined mesh (i.e., the $5.7 \times 10^{6}$ elements grid).

From the pressure drops summarized in Table 1, it was observed that the $2.7 \times 10^{6}$ elements grid provided accurate predictions regarding the most refined mesh, with a smaller computational cost. In this context, this mesh refinement was defined as independent and used in further numerical analysis. The spatial parameters used in the grid generation of the $2.7 \times 10^{6}$ elements grid were employed in the spatial discretization of all other geometry designs. 
Table 1. Pressure drops predicted in the independence mesh test for $R e=100$.

\begin{tabular}{lll}
\hline Mesh Refinement & Pressure drop, $\Delta \mathrm{P}$ in MPa & Relative deviation ${ }^{\text {a) }}(\%)$ \\
\hline $1 \times 10^{6}$ & 35.769 & 18.303 \\
$2 \times 10^{6}$ & 37.926 & 13.377 \\
$2.7 \times 10^{6}$ & 43.640 & 0.326 \\
$3.7 \times 10^{6}$ & 44.101 & -0.726 \\
$4.6 \times 10^{6}$ & 45.462 & -3.834 \\
$5.7 \times 10^{6}$ & 43.783 & -- \\
\hline
\end{tabular}

a) Deviation from the most refined grid $\left(5.7 \times 10^{6}\right.$ elements)

\subsection{Design Performance: Fluid Mixing Analysis}

The performance of the four proposed designs was assessed by fluid mixing under the Reynolds number range of 0.01-100 for vegetable oil-ethanol flow. Figure 3 presents the mixing indexes obtained for all three channel heights. Figure 4 presents the ethanol distribution along the channel for $R e=0.1$ for all three channel heights.
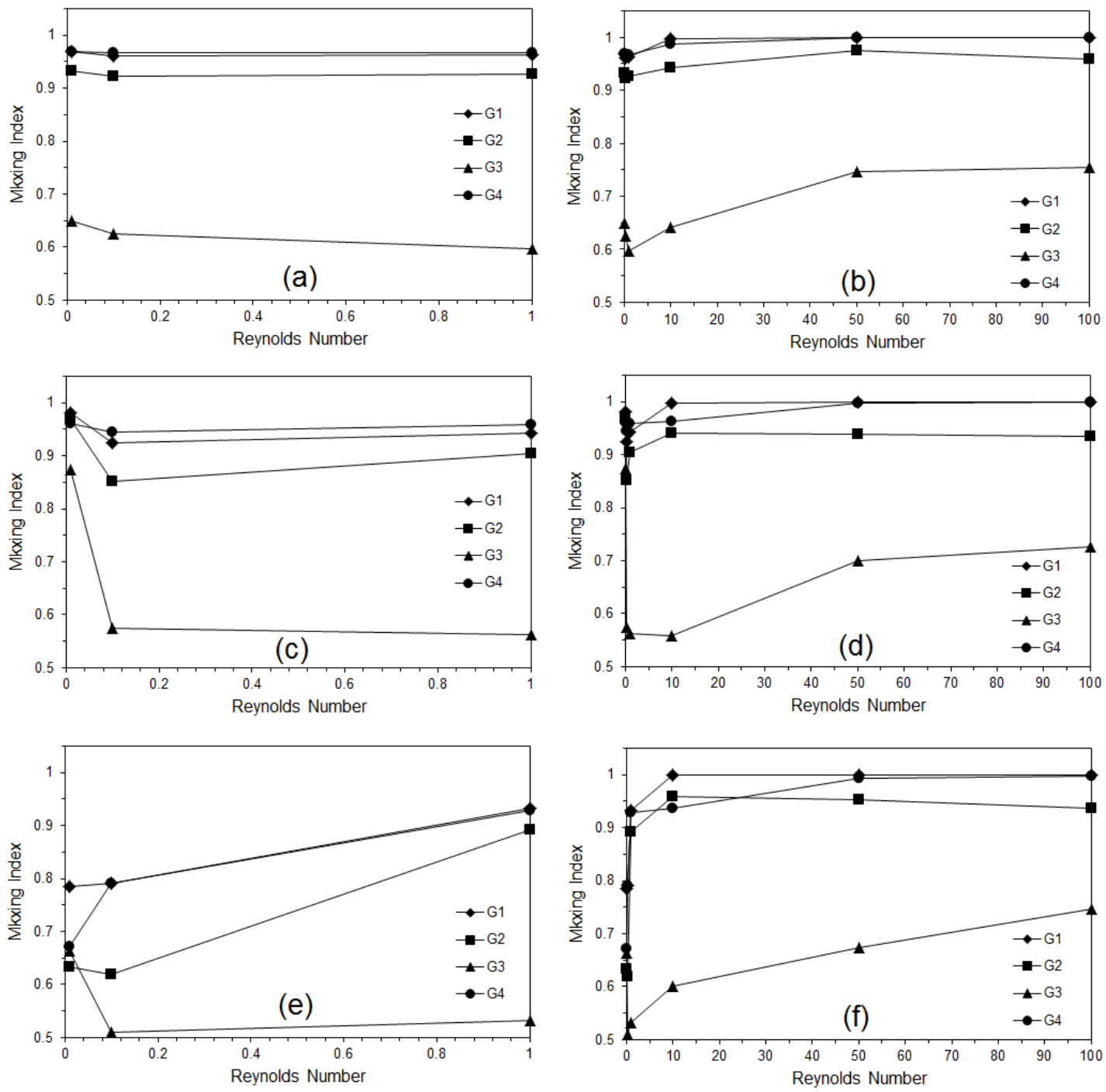

Figure 3. Mixing indexes obtained for geometries G1-G4, operating under the Reynolds number range from 0.01 to 100, with different channel heights, $H$ : (a) $200 \mu \mathrm{m}$ (Re range: 0.01-1); (b) $200 \mu \mathrm{m}$ (Re range: 
0.01-100); (c) $500 \mu \mathrm{m}$ (Re range: 0.01-1); (d) $500 \mu \mathrm{m}$ (Re range: 0.01-100); (e) $1000 \mu \mathrm{m}$ (Re range: 0.01-1); (f) $1000 \mu \mathrm{m}$ (Re range: 0.01-100).

For all three channel heights the inferior performance was noticed for $\mathrm{G} 3$ design, resulting in mixing indexes below 0.75 (Figure 3). Geometries G1 and G4 exhibited superior performances, with high mixing index in high Reynolds number $(R e>10$ ) (Figure $3 b, \mathrm{~d}, \mathrm{f})$. The $\mathrm{G} 2$ design provided high mixing index about 0.95 for the higher Reynolds number range. The channel height effects on mixing process were more pronounced at very low Reynolds numbers, as detailed in Figure 3a,c,e. At $\operatorname{Re}=0.01$, high mixing was achieved for $H=200 \mu \mathrm{m}$ (Figure 3a) for geometries G1, G2 and G4. The mixing efficiency decreased with the increment of channel height to $1000 \mu \mathrm{m}$ (Figure 3e). These results can be attributed to the molecular diffusion mechanism, as illustrated by Figure 4 .

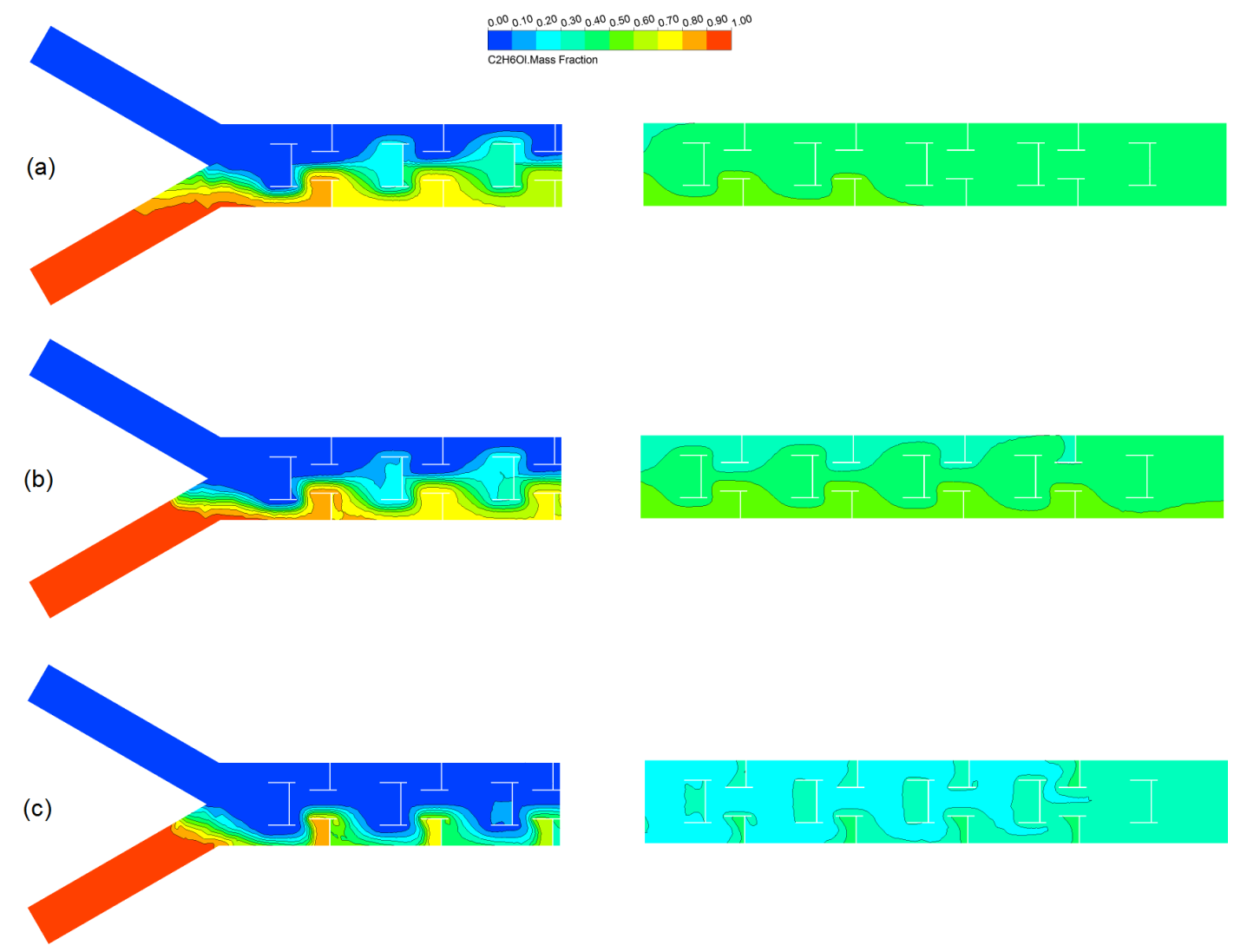

Figure 4. Ethanol mass fraction contours at a mid-plane in $\mathrm{G} 1$ device entrance region (left) and outlet section (right) at Re $=0.1$ for channel heights of $H$ : (a) $200 \mu \mathrm{m}$; (b) $500 \mu \mathrm{m}$, (c) $1000 \mu \mathrm{m}$.

From Figure 4 the effect of scale-up on the mixing performance can be highlighted for $R e=0.1$, operating under pure diffusive mass transport. For the channel height of $200 \mu \mathrm{m}$ (Figure 4a), a quasicomplete mixing between oil and ethanol was observed in the outlet zone $(M=0.960)$, being complete about the third micromixer unit from the channel outlet. The increment of channel height to $500 \mu \mathrm{m}$ (Figure $4 \mathrm{~b}$ ) resulted in two mass fraction contours at channel outlet, characterizing a decrease on mixing degree $(M=0.925)$, as observed in Figure 3c. Further channel height increment also affected negatively the mixing process and different concentration zones were observed in micromixer units located at outlet zone, mostly in stagnant zone of the static mixer. Accordingly, the mixing index decreased from 0.960 to 0.791 with the increment of the channel height from $200 \mu \mathrm{m}$ to $1000 \mu \mathrm{m}$ for $R e=0.1$ in the geometry G1.

Also, the increment of $R e$ from 0.01 to 0.1 affected negatively the mixing process in the microchannels with $H=500 \mu \mathrm{m}$ and $1000 \mu \mathrm{m}$ (Figures 3c,e). This behavior can be attributed to the reduction of fluid residence time, resulting in an ineffective diffusion mass transfer, i.e., the required time to complete mixing between oil and ethanol were not sufficient. Considering the proposed design effects 
on mixing, geometries $\mathrm{G} 1$ and $\mathrm{G} 4$ exhibited superior performance with a lower mixing efficiency reduction with scaling-up regarding G2 and G3 devices. Increasing the Reynolds number from 10, chaotic advection mechanism starts to be effective, as shown in Figure 5 by the velocity vector fields obtained in geometry $\mathrm{G} 4$, overcoming the shortage of residence time to complete the mixing process by molecular diffusion, and consequently, the mixing index increased again.

Higher Reynolds numbers denote high throughput of the microdevice, however, the pressure drop also increase. Figure 6 presents the pressure drops and the performance indexes, i.e., the mixing efficiency per unit pressure drop. As expected, operation under very low Reynolds numbers resulted in higher performance indexes due to the almost negligible pressure drops. Geometry designs $\mathrm{G} 2$ and G3 exhibited superior performance indexes, with magnitude orders about $10^{2}$ greater than G1 and G4 (Figure $6 a, c, e)$, indicating that to provide superior mixing, the geometries $\mathrm{G} 1$ and $\mathrm{G} 4$ required higher pressure drops, as observed in Figure 6b,d,f.

As expected, small micrometric channel requires very high pressure drops to achieve relative high Reynolds numbers $(R e \geq 50)$. Since the flow is characterized by laminar flow regime, the pressure drop increased almost linearly with the Reynolds number increment. The G2 and G3 designs exhibited the lower pressure drops, about $35 \mathrm{MPa}$ for $H=200 \mu \mathrm{m}$, decreasing to less than $3.1 \mathrm{MPa}$ for $H=1000 \mu \mathrm{m}$. The higher pressure drops resulting from $\mathrm{G} 1$ and $\mathrm{G} 4$ devices can be attributed to the chaotic advection generated from the static mixer designs, as observed in Figure 7. In general, for $\operatorname{Re} \geq 10$, recirculation zones appears in geometries G1 and G4 (Figure 7a,d). In contrast, G2 and G3 layouts did not induced chaotic advection (Figure $7 b, c$ ), promoting a maximum effect of changing the stream direction, but not generating vortex patterns. Accordingly, the mixing performance was inferior for $\mathrm{G} 2$ and $\mathrm{G} 3$, however, a lower pressure drop was also observed in such devices. Comparing the pressure drop of $\mathrm{G} 1$ and $\mathrm{G} 4$, the latter one resulted in a very high head loss, once the flow pattern presented more recirculation zones in G4 (Figure 7a,d). Geometry G1 presented a relative moderate pressure drop of $7.73 \mathrm{MPa}$ for $H=1000 \mu \mathrm{m}$, concerning the $96 \mathrm{MPa}$ of pressure drop for $H=200 \mu \mathrm{m}$. 
(a) $\operatorname{Re}=0.01$

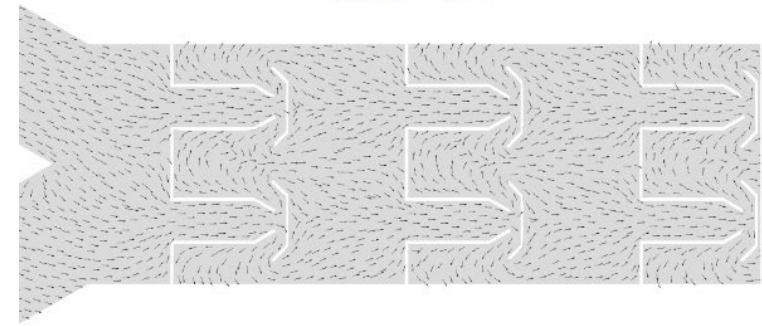

(c) $\operatorname{Re}=1$

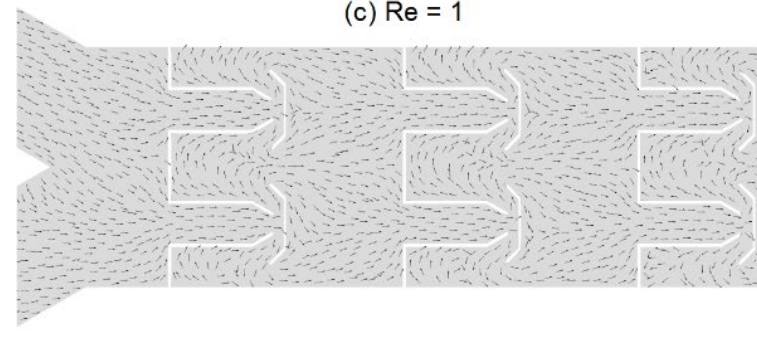

(e) $\operatorname{Re}=50$

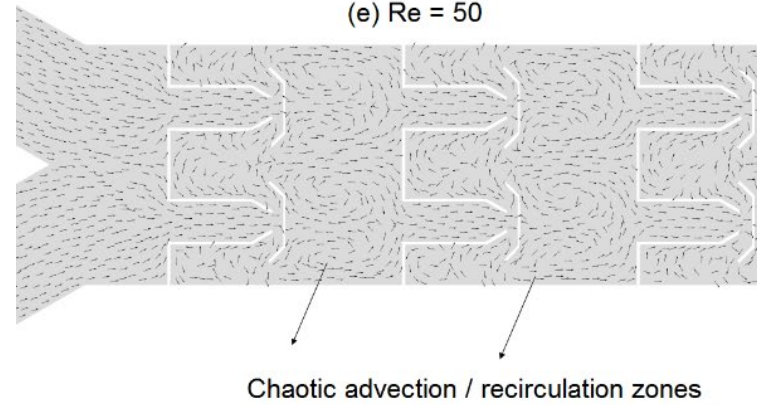

(b) $\operatorname{Re}=0.1$

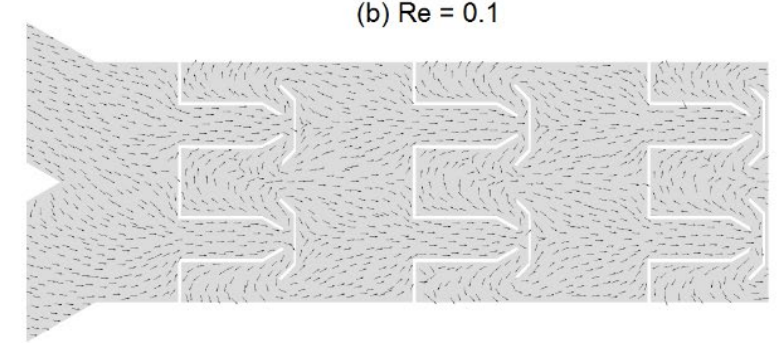

(d) $\operatorname{Re}=10$

(f) $\operatorname{Re}=100$

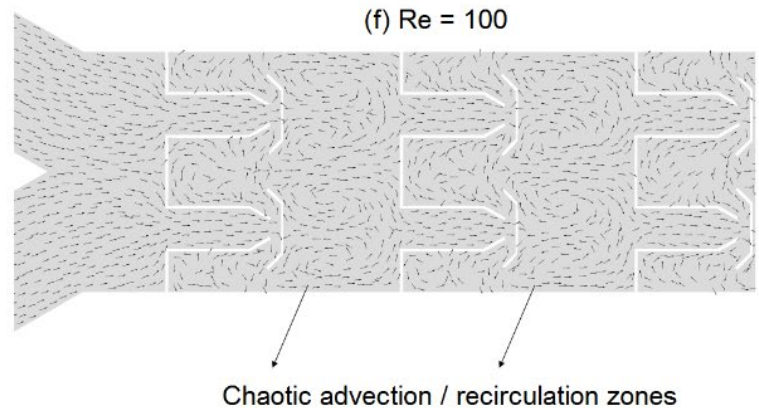

Figure 5. Velocity vector field (mid-plane) obtained for the first three micromixer units for geometry G4 with $H=200 \mu \mathrm{m}$ at Reynolds numbers of: (a) 0.01 ; (b) 0.1 ; (c) 1 ; (d) 10; (e) 50 ; (f) 100 ; and details of chaotic advection / recirculation flow patterns observed for $R e \geq 10$. 

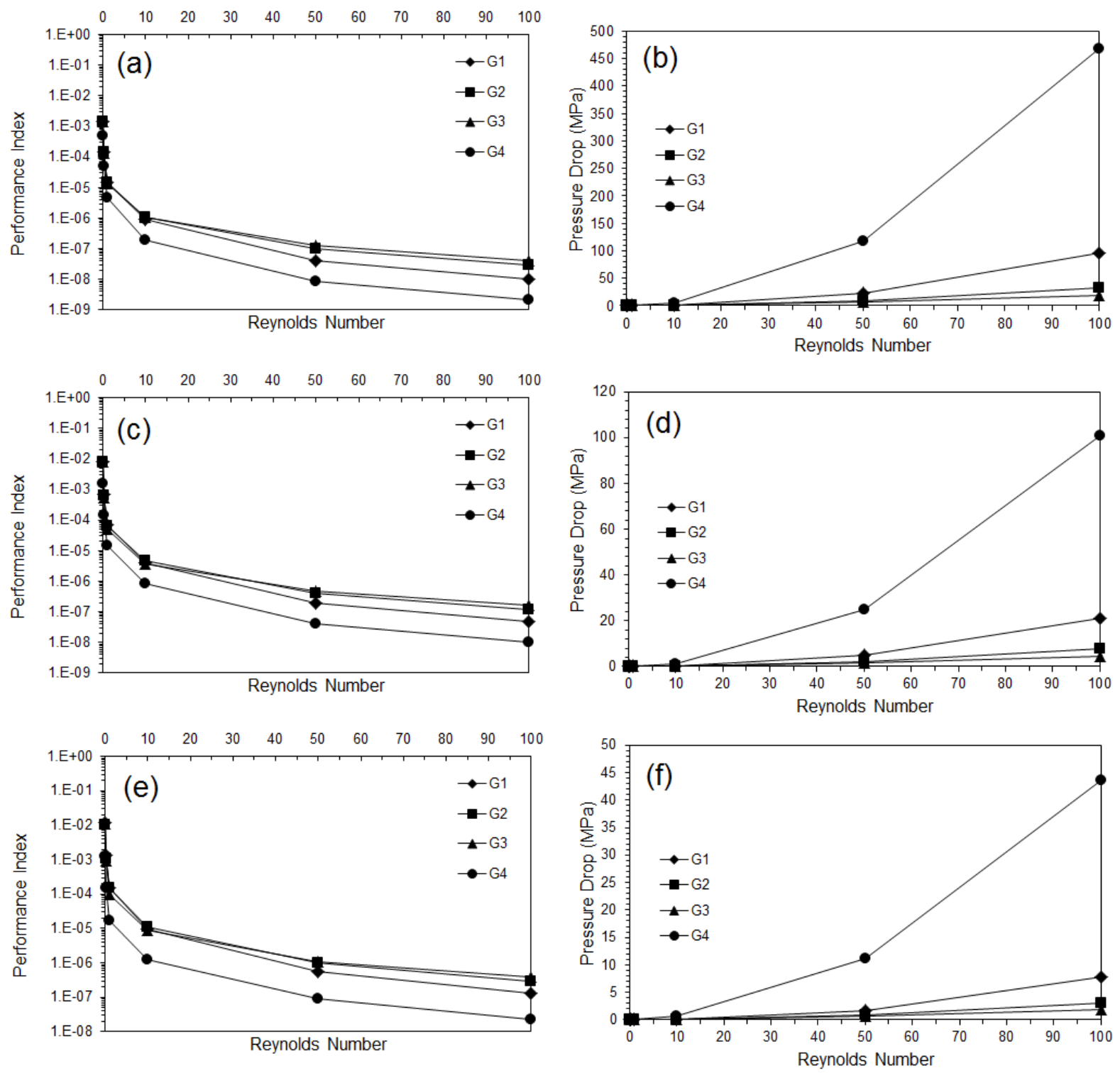

Figure 6. Performance index (in \% $\mathrm{Pa}^{-1}$ ) observed for geometries G1-G4, operating under the Reynolds number range from 0.01 to 100, with different channel heights, $H$ : (a) $200 \mu \mathrm{m}$; (c) $500 \mu \mathrm{m}$; (e) $1000 \mu \mathrm{m}$; Pressure drops predicted in the geometries G1-G4, operating under the Reynolds number range from 0.01 to 100 , with different channel heights, $H$ : (b) $200 \mu \mathrm{m}$; (d) $500 \mu \mathrm{m}$; (f) $1000 \mu \mathrm{m}$. 


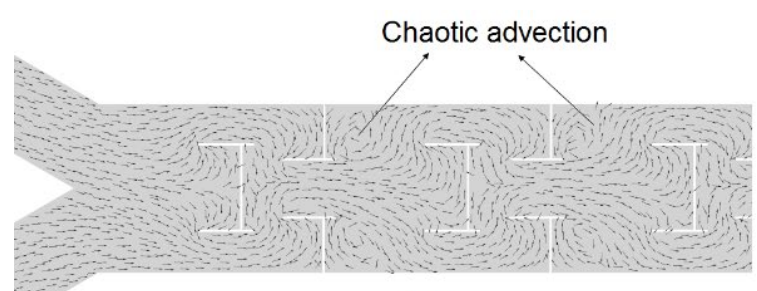

(a)

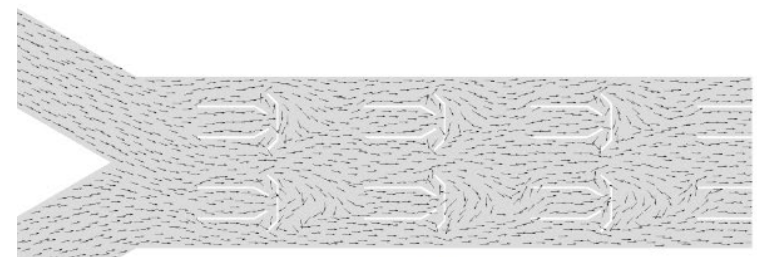

(c)

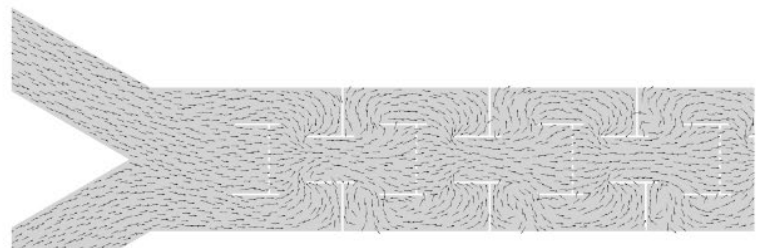

(b)

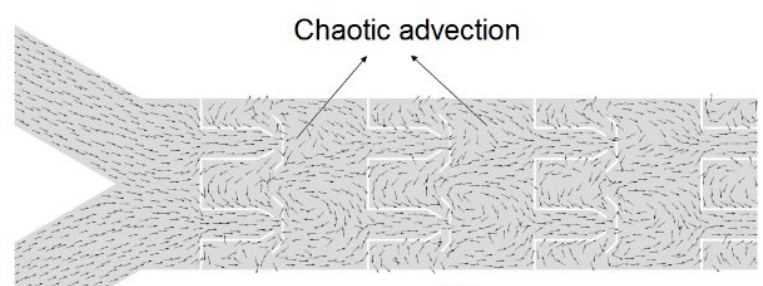

(d)

Figure 8. Velocity vector field (mid-plane) obtained for $H=200 \mu \mathrm{m}$ at $R e=10$, for geometry designs: (a) G1; (b) G2; (c) G3; (d) G4; and details of chaotic advection / recirculation flow patterns observed in G1 and $\mathrm{G} 4$.

Observing the velocity vector fields shown in Figure 7, the static mixer designs proposed in G1 and $\mathrm{G} 4$ were efficient to induce recirculation zones at $R e=10$. Further increment on fluid velocity intensifies the chaotic advection mechanism, promoting faster mixing between the chemical species. Figure 8 shows the velocity vector fields combined with the ethanol mass fraction distribution for all geometries at $R e=$ 100 , i.e., the highest flow rate evaluated. The macroscopic mixing induced by G1 design was the most effective (Figure 8a), once after four micromixer units the mixing was almost complete (Figure 9a).

The proposed layout promoted a chaotic advection mechanism with a progressive macroscopic transport along the micromixer units, resulting in relative moderate pressure drops, as discussed ahead and shown in Figure 9b. Static mixers of G2 (Figure 8b) and G3 (Figure 8c) barely disturbed the flow even at $R e=100 . G 2$ design promoted recirculation zones only at channel sides, where the fluids are almost pure due to the Y-shape feed (Figure 8b). Geometry $\mathrm{G} 3$ generated more vortex zones (Figure 8c) regarding the G2, however, the flow pattern was not so effective compared to G1 or G4. The G4 layout induced strong recirculation zones at a very high pressure drop cost. In order to evaluated the devices global performance, mixing performance and pressure drop as a function of micromixer units was evaluated as shown in Figure 9. 
(a)
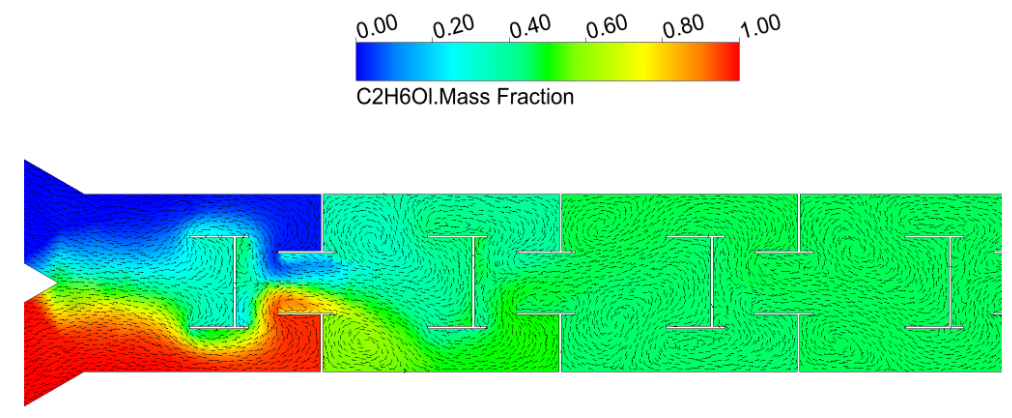

(b)

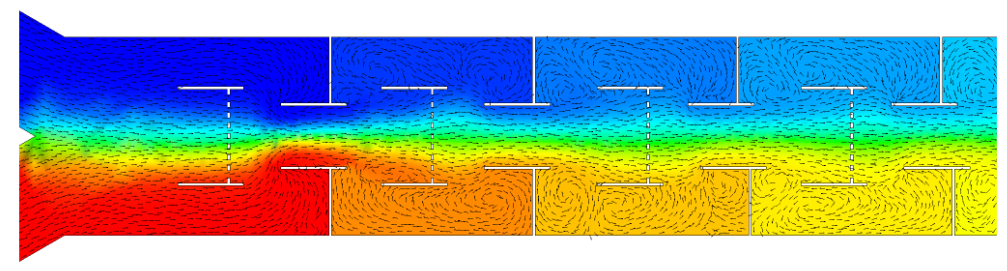

(c)

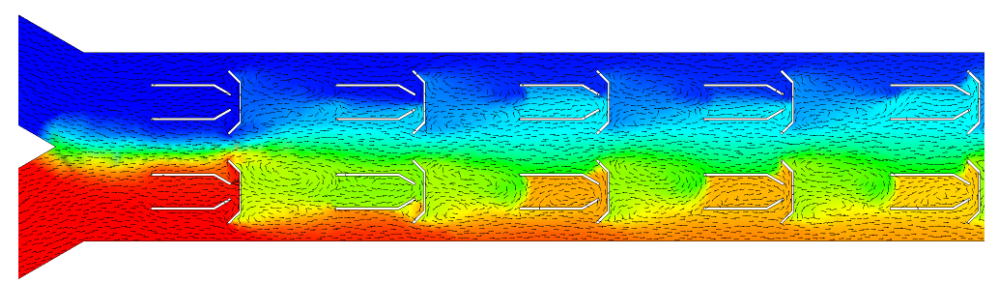

(d)

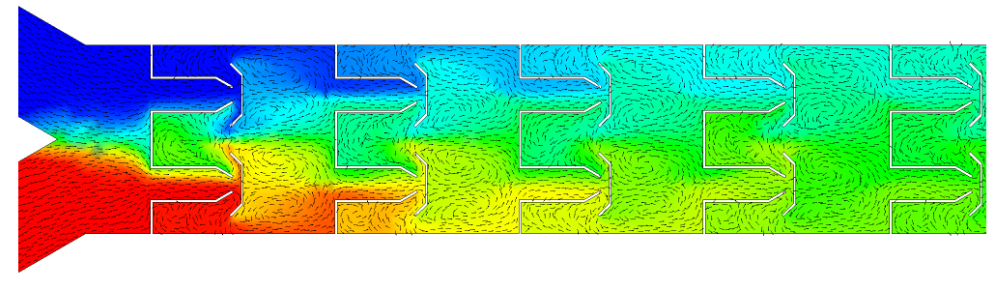

Figure 8. Ethanol mass fraction distribution and velocity vector fields at a mid-plane for geometries: (a) G1; (b) G2; (c) G3; (d) G4 (Re=100, $H=200 \mu \mathrm{m})$.
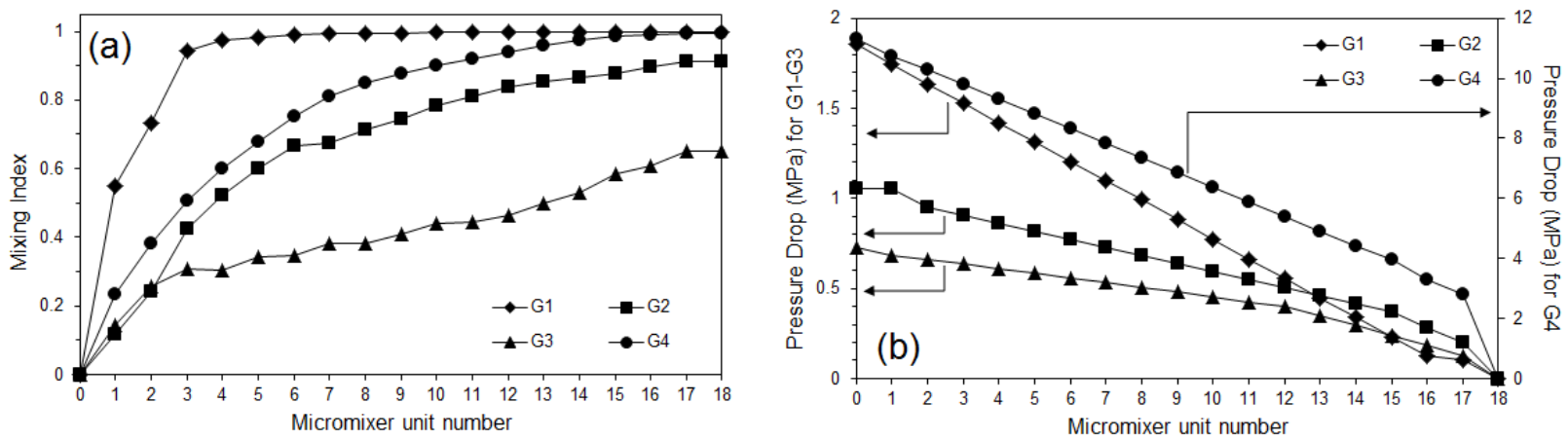

Figure 9. Devices performance along the micromixer units for $R e=50$ and $H=1000 \mu \mathrm{m}$ : (a) Mixing index; (b) Pressure drop (Micromixer unit number of 0 and 18 indicate inlet and outlet, respectively).

From Figure 9a was noticed that G1 design promoted an almost complete mixing after only four micromixer units for $R e=50$, with a respective pressure drop about $0.5 \mathrm{MPa}$ (difference between inlet and micromixer unit pressure drops, Figure $9 \mathrm{~b}$ ). Further increment of micromixer units maintained the mixing degree, since it already achieved $M>0.975$, i.e., the device $\mathrm{G} 1$ can be operated with about 4-5 micromixer units, resulting in high mixing degree with relative low pressure drop, with an operational flow 
rate about $0.21 \mathrm{~L} / \mathrm{min}$. Geometries $\mathrm{G} 2$ and $\mathrm{G} 3$ presented low relative pressure drops, however, required all 17 micromixer units to achieve an incomplete mixing, about $M=0.912$ (G2) and $M=0.651$ (G3), that can be an issue in mass-transfer limiting process, e.g., the incomplete consumption of the limiting reactant becoming the process ineffective. The geometry $G 4$ achieved high mixing indexes $(M>0.975)$ with more than 14 micromixer units, however, the pressure drop was very high, about $7 \mathrm{MPa}$, regarding G1 that for a same geometric configuration (14 micromixer units) required a pressure drop of $1.5 \mathrm{MPa}$.

\section{Conclusions}

Four geometry designs were proposed based on distillation tray layouts aiming the use as micromixers for scale-up. The mixing performance was evaluated by numerical simulation using CFD for a Reynolds number range of 0.01-100, encompassing diffusive and convective mass transfer regimes. The mixing between vegetable oil and ethanol and the pressure drop were assessed. The effect of scale-up was also investigated by changing channel heights from 200, 500 and $1000 \mu \mathrm{m}$. At $R e=0.01$, the fluids experiences sufficient time for an almost complete mixing by diffusive transport, resulting in high mixing indexes for geometries $\mathrm{G} 1, \mathrm{G} 2$ and $\mathrm{G} 4$ for $H=200 \mu \mathrm{m}$, however with a very low throughput. The scale-up, i.e., the increment of $\mathrm{H}$, resulted in decrease on mixing performance. Also, the increment of Re from 0.01 to 0.1 reduced the mixing degree, due to the reduction on fluid residence time to complete the mass transfer by pure diffusion process. Further increment in Reynolds number resulted in induction of recirculation patterns, i.e., macroscopic or convective contribution to mass transfer, mostly for $\mathrm{Re}=10$ or above. Geometries $\mathrm{G} 1$ and $\mathrm{G} 4$ generated effective chaotic advection from $R e=10$, resulting in good mixing performance, in contrast, higher pressure drops were observed. Geometry G1 achieved high mixing index, above 0.975 , with only four micromixer units and a pressure drop about $0.5 \mathrm{MPa}$. In general, considering the aforementioned, the geometry $\mathrm{G} 1$ with a height of $H=1000 \mu \mathrm{m}$ exhibited a good performance, i.e., high mixing degree between fluids, with a reasonable pressure drop, showing great potential to be used in microdevices scale-up and numbering-up for modular chemical plants application. The good performance can be attributed to the chaotic advection mechanism induced by the G1 design, promoting a high and progressive recirculation that increased the macroscopic mixing with a relative moderate pressure drop.

\section{Acknowledgment}

The authors would like to thank the National Postdoctoral Program (PNPD/Capes), the Unicamp Scholarship Program, FUNCAMP (Unicamp Foundation) and the financial support provided by CNPq (National Council for Scientific and Technological Development, Process 404760/2016-3), FAPESP (São Paulo Research Foundation, Process 2013/25850-7 and Process 2016/ 20842-4) and FAPEMIG (Minas Gerais Research Foundation, Process APQ-02144-17). This study was financed in part by the Coordenação de Aperfeiçoamento de Pessoal de Nível Superior - Brasil (CAPES) - Finance Code 001.

\section{Symbols used}

Greek letters

$\begin{array}{lll}\rho & {\left[\mathrm{kg} \mathrm{m}^{-3}\right]} & \text { specific mass } \\ \sigma & {[-]} & \text { mass fraction variance } \\ \mu & {\left[\mathrm{kg} \mathrm{m}^{-1} \mathrm{~s}^{-1}\right]} & \text { dynamic viscosity }\end{array}$

\section{Abbreviations}

$\begin{array}{lll}D_{A B} & {\left[\mathrm{~m}^{2} \mathrm{~s}^{-1}\right]} & \text { mass diffusion coefficient } \\ D_{h} & {[\mathrm{~m}]} & \text { hydraulic diameter } \\ g & {\left[\mathrm{~m} \mathrm{~s}^{-2}\right]} & \text { gravity acceleration constant }\end{array}$


Peer-reviewed version available at Chemical Engineering \& Technology 2020; doi:10.1002/ceat.201900668

\begin{tabular}{|c|c|c|}
\hline$H$ & {$[\mathrm{~m}]$} & channel height \\
\hline$L_{c}$ & {$[\mathrm{~m}]$} & characteristic length \\
\hline$L_{h}$ & {$[m]$} & characteristic length in geometry $G 2$ \\
\hline$M$ & {$[-]$} & mixing index \\
\hline$N$ & {$[-]$} & number of sampling points \\
\hline$P$ & {$\left[k g \mathrm{~m}^{-1} \mathrm{~s}^{-2}\right]$} & pressure \\
\hline$P I$ & {$\left[\% \mathrm{~Pa}^{-1}\right]$} & performance index \\
\hline$U$ & {$\left[m s^{-1}\right]$} & velocity \\
\hline$Y_{A}$ & {$[-]$} & mass fraction of chemical species $A$ \\
\hline$Y_{B}$ & {$[-]$} & mass fraction of chemical species $B$ \\
\hline$Y_{i}$ & {$[-]$} & mass fraction of sample point $i$ \\
\hline
\end{tabular}

\section{References}

[1] T. Wirth, Microreactors in organic chemistry and catalysis. Wiley-VCH Verlag GmbH \& Co., Weinheim, German John Wiley \& Sons, 2013.

[2] A. Pohar, I.Plazl, Chem. Biochem. Eng. Quat., 2009, 23, 537-544. DOI: 10.15255/CABEQ.2014.284

[3] G.M. Whitesides, Nature, 2006, 442, 368-373. DOI: 10.1038/nature05058

[4] S. Neethirajan, I. Kobayashi, M. Nakajima, D. Wu, S. Nandagopal, F. Lin, Lab Chip, 2011, 11, 15741586. DOI: $10.1039 /$ COLCO0230E

[5] B. Gumuscu, J.G. Bomer, H.L. De Boer, A. Van Den Berg, J.C., Eijkel, Microsyst. Nanoeng., 2017, 3, 17001. DOI: $10.1038 /$ micronano.2017.1

[6] T.A. Balbino, J.M. Serafin, A.A.M. Gasperini, C.L.P., De Oliveira, L.P. Cavalcanti, M.B., De Jesus, L.G. De La Torre, Langmuir, 2016, 32, 1799-1807. DOI: 10.1021/acs.langmuir.5b04177

[7] V. Vaghi, C. Potrich, L. Pasquardini, L. Lunelli, L. Vanzetti, E. Ebranati, A. Lai, G. Zehender, D. Mombello, M. Cocuzza, C.F. Pirri, C. Pederzolli, Biophys. Chem., 2016, 208, 54-61. DOI: 10.1016/j.bpc.2015.06.005

[8] J. Chen, D. Chen, T. Yuan, X. Chen, Y. Xie, H. Fu, D. Cui, X. Fan, M.K.K. Oo, Microelectron. Eng., 2014, 128, 36-41. DOI: 10.1016/j.mee.2014.05.032

[9] J.L. Silva Jr., H.S. Santana, in Process Analysis, Design, and Intensification in Microfluidics and Chemical Engineering, $1^{\text {st }}$. ed. (Eds: H.S. Santana, J.L. Silva Jr, O.P. Taranto), IGI Global, Hershey, PA, USA, 2019, Ch. 6.

[10] A.F. Al-Neama, N. Kapur, J. Summers, H.M. Thompson, Appl. Therm. Eng., 2017, 116, 709-723. DOI: 10.1016/i.applthermaleng.2017.02.001

[11] H.S. Santana , G.B., Sanchez, O.P. Taranto. Chem. Eng. Res. Des., 2017, 124, 20-28, DOI: 10.1016/i.cherd.2017.05.022

[12] J. Li, G.P. Peterson, P. Cheng. Int. J. Heat Mass Tran., 2004, 47, 4215-4231, DOI: 10.1016/j.ijheatmasstransfer.2004.04.018

[13] C.P. Lee, M.F., Lai. J. Appl. Phys., 2010, 107, 09B524. DOI: $10.1063 / 1.3358615$ 
[14] H.S. Santana, D.S. Tortola, É.M. Reis, J.L., Silva, O.P. Taranto, Chem. Eng. J., 2016, 302, $752-762$. DOI: $10.1016 /$ j.cej.2016.05.122

[15] P. Sobieszuk, F. Ilnicki, R. Pohorecki. Chem. Proc. Eng., 2014, 35(1), 35-45, DOI: 10.2478/cpe-20140003

[16] P. Watts, C. Wiles, Org. Biomol. Chem., 2007, 5, 727-732. DOI: 10.1039/B617327F

[17] M.P. Marques, P. Fernandfes, Molecules, 2011, 16, 8368-8401. DOI: 10.3390/molecules16108368.

[18] N. Wang, X. Zhang, B. Chen, W. Song, N.Y., Chan, H.L.W. Chan. Lab Chip, 2012, 12, 3983-3990, DOI: 10.1039/C2LC40428A

[19] H.S. Santana , J.L., Silva, O.P. Taranto, Sensor. Actuat. B-Chem., 2019, 281, 191-203. DOI: 10.1016/j.snb.2018.10.089

[20] H.S. Santana, D.S. Tortola, J.L., Silva Jr., O.P. Taranto, Energ. Conv. Manage., 2017, 141, $28-39$. DOI: $10.1016 /$ j.enconman.2016.03.089

[21] N.T. Nguyen, S.T. Wereley. Fundamentals and Applications of Microfluidics. Publishing House Artech House, Boston/London, 2006.

[22] C.Y. Lee, W.T. Wang, C.C. Liu, L.M. Fu. Chem. Eng. J., 2016, 288, 146-160, DOI: 10.1016/j.cej.2015.10.122

[23] V. Hessel, H. Löwe, F. Schönfeld, Chem. Eng. Sci., 2005, 60, 2479-2501, DOI: 10.1016/j.ces.2004.11.033 